\title{
Precision Electroweak Constraints on Near-Conformal Dynamical EWSB
}

\section{Luigi Del Debbio}

SUPA, School of Physics and Astronomy, University of Edinburgh

Edinburgh EH9 3JZ, UK

E-mail: luigi.del.debbio@ed.ac.uk

\section{Eoin Kerrane*}

Instituto de Física Téorica UAM/CSIC

Universidad Autónoma de Madrid

Cantoblanco E-28049 Madrid, Spain

E-mail: eoin.kerrane@csic.es

\begin{abstract}
Early attempts to construct models of Dynamical Electro-Weak Symmetry Breaking (DEWSB), assuming familiar dynamics for the technisector, failed to overcome strict constraints from the oblique correction parameter S. More recently it has been suggested that models involving a technisector with near-conformal dynamics might be more successful in avoiding these constraints. In such theories the reduced strength of chiral symmetry breaking is expected to suppress the splitting between the vector and axial channels, leading to a reduced contribution to $S$.

We describe an ongoing attempt to measure the contribution to the S parameter from a technisector involving the $S U(2)$ theory with two flavours of adjoint Dirac fermions, which is the main component of the minimal walking-technicolor (MWT) model. The dynamics of this theory have been extensively studied and it has been shown to be consistent with the presence of an infra-red conformal fixed point.

We study the S parameter in this theory using a mixed-action lattice simulation, using Wilson sea fermions and Domain-Wall fermions in the valence sector. We describe the steps involved in the computation, explore important systematic effects, and present current results.
\end{abstract}

The 30th International Symposium on Lattice Field Theory

June 24 - 29, 2012

Cairns, Australia

\footnotetext{
*Speaker.
} 


\section{Introduction}

One possible explanation for the origin of Electroweak Symmetry Breaking (EWSB) is that it occurs Dynamically (DEWSB) as a consequence of the spontaneous breaking of the chiral symmetry of new fermionic matter fields at energies below the electroweak scale due to the strongly coupled nature of a new gauge interaction at these energies. In its simplest incarnation, this idea, generally referred to as Technicolor, encounters difficulties in remaining compatible with current experimental constraints. The most fundamental of these difficulties is the tension with electroweak precision results generated when new fermionic degrees of freedom are added to the theory.

In particular the $\mathrm{S}$ parameter is sensitive to the amount of matter content in the theory. Indeed, current bounds on the S parameter strongly disfavour the existence of a fourth generation of degenerate fermions. The introduction of additional matter fields in a Technicolor model can generate a contribution to the $\mathrm{S}$ parameter which are in conflict with the experimental bound.

More recent Technicolor models rely on the new gauge interactions being such that their dynamics are markedly different from those of QCD. If the additional gauge sector has a nearconformal dynamics it is expected to enable such models to avoid difficulties with constraints on flavour-changing interactions encountered when it is attempted to extend the EWSB mechanism to generate fermion masses. It is also suggested that such dynamics would reduce the contribution to the $S$ parameter from the additional matters in the Technicolor model. Estimating the $S$ parameter contribution from models of DEWSB is inherently difficult due to the importance of the non-perturbative physics of the model at low energies, and the deviation of the dynamics of the interactions involved from the familiar dynamics of QCD.

Lattice simulations provide a valid first-principles, fully non-perturbative approach to such strongly coupled theories, and a calculation of the S parameter theory from a give gauge sector is possible. Such calculations have been done for QCD [1,2] and for the $S U(3)$ gauge theory with additional fundamental fermions.

One of the interesting candidates for the Technisector of a model of DEWSB is the $S U(2)$ gauge theory with two flavours of Dirac fermion in the adjoint representation, which has been coined Minimal Walking Technicolor (MWT). This theory has been studied in detail and all evidence suggests that this theory lies within the conformal window, and so will flow to a conformal fixed point in the infra-red. In this article we present a calculation of the contribution to the $S$ parameter generated when this gauge sector is added to the Standard Model in a DEWSB model.

The $\mathrm{S}$ parameter is one of a group of quantities introduced to parameterise quantum corrections to electroweak observables [3, 4]. They are built from the currents $J_{\mathrm{X}}^{\mu}(x)$ where the placeholder $\mathrm{X}$ is one of $\{1,2,3, Q\}$ denoting the components of the weak-isospin currents and the electromagnetic current. From these the vacuum polarisation functions $\Pi_{\mathrm{XY}}^{\mu v}$ are defined as

$$
\begin{aligned}
i \Pi_{\mathrm{XY}}^{\mu v}(q)=i \int d^{3} x\left\langle J_{\mathrm{X}}^{\mu}(x) J_{\mathrm{Y}}^{v}(0)\right\rangle & =\mathrm{X}, \mu \sim \mathrm{Y}, v \\
& =i g^{\mu v}\left(\Pi_{X Y}(0)+q^{2} \Pi_{X Y}^{\prime}\left(q^{2}\right)\right)+\left(\sim q^{\mu} q^{v}\right)
\end{aligned}
$$


and from these the parameters $S, T$ and $U$ are defined. Specifically

$$
S=16 \pi\left[\Pi_{33}^{\prime}(0)-\Pi_{3 Q}^{\prime}(0)\right] .
$$

Assuming conservation of iso-spin and parity

$$
\Pi_{33}=\frac{1}{4}\left(\Pi_{\mathrm{Vv}}+\Pi_{\mathrm{AA}}\right) \quad \Pi_{3 \mathrm{Q}}=\frac{1}{2} \Pi_{\mathrm{VV}}
$$

which allows us to re-express $S$ as

$$
S=-4 \pi\left[\Pi_{\mathrm{VV}}^{\prime}(0)-\Pi_{\mathrm{AA}}^{\prime}(0)\right]
$$

It is this expression which allows us to directly calculate $S$ on the lattice. Despite this definition of the S parameter, when discussing experimental bounds on its value, what is conventionally quoted is the difference between the measured value, and the expected theoretical value as predicted in the Standard Model at a certain reference Higgs mass. With this definition, the current bound is $S=0.03 \pm 0.09[5]$.

There are a number of estimates of the $\mathrm{S}$ parameter contribution in different models. The original authors made a number of estimates [3, 4]; to first order in perturbation theory, $N_{\mathrm{f}}$ flavours of fermion in a representation $R$ give a contribution

$$
S \simeq \frac{1}{6 \pi}\left(\frac{N_{\mathrm{f}} d(R)}{2}\right)
$$

where $d(R)$ is the dimension of the representation $R$. They also attempted a semi-phenomenological estimate evaluating a dispersive integral expression for $S$ using existing QCD data, which led them to numerical estimates which are well approximated by the expression

$$
S \simeq 0.3 \frac{N_{\mathrm{f}}}{2} \frac{d(R)}{3}
$$

where we have inserted the same scaling with the representation of the fermions as in the perturbative expression.

These estimates, however, cannot be simply applied to any gauge sector as they will not account for effects of strongly-coupled physics or deviation of the gauge dynamics from those of QCD. There have been estimates of the $S$ parameter contribution in models of interest for DEWSB, in particular near-conformal models, which suggest that the contribution may be suppressed in such theories [6]. Previous lattice estimates of the $S$ parameter contribution from near-conformal models are restricted to a calculation in the $S U(3)$ theory with a large number of fundamental fermions [7].

\section{MWT Calculation}

We present a calculation of the $S$ parameter contribution generated when the $S U(2)$ gauge theory with 2 flavours of fermion in the adjoint representation is incorporated in a model of DEWSB. This calculation builds on simulations of the MWT theory used to study the spectroscopy and other aspects of the theory [8]. The ensembles of gauge configurations were generated using the Wilson fermion discretisation at a single gauge coupling $\beta=2.25$, and we study ensembles with two volumes $\left(32 \times 16^{3}\right.$ and $\left.64 \times 24^{3}\right)$ and several fermion masses approaching the chiral limit. 
As we will compute the $S$ parameter in this theory via the difference between the vector and axial channels (1.3), we will require a well conserved chiral symmetry in our lattice calculation. As a result, using the Wilson fermion formulation in both the sea and valence sectors will not suffice. We must conduct a mixed-action computation, using a chiral valence action.

For this we choose the Domain Wall Fermion (DWF) action for the valence sector. To do this we must tune the bare DWF fermion mass $m_{\mathrm{f}}$ to reproduce the physics as measured in the unitary Wilson calculation. We do this primarily via the effective pseudoscalar mass $m_{\mathrm{PS}}(t)$. An example of this tuning is shown in Fig. 1a. The bare fermion mass $m_{\mathrm{f}}$ at which the tuning is achieved is found to be largely independent of the volume in question, and so we are able to perform the tuning on the smaller volume and use the value obtained in computations on the larger volume.

We monitor the degree to which the chiral symmetry is explicitly broken in our calculation by measuring the residual mass $m_{\text {res }}$ [9], defined through the pseudoscalar currents on the boundary $J_{5}$ and at the center $J_{5 \mathrm{q}}$ of the fifth dimension, which has extent $L_{5}$. The residual mass is expected to decay as $L_{5}$ is increased, and the vanishing of $m_{\text {res }}$ in the limit of infinite $L_{5}$ restores the explicit chiral symmetry.

At present all data in this study are computed at $L_{5}=16$. To achieve a reasonable degree of chiral symmetry preservation in our calculation we would like maintain a ratio $\frac{m_{\mathrm{res}}}{m} \ll 1$. In Fig. $1 \mathrm{~b}$ we see that the ratio of $m_{\text {res }}$ to the quark mass $m$ grows in the chiral limit, meaning the effect of the chiral symmetry breaking on the physics of interest will be enhanced as we reduce the quark mass, keeping $L_{5}$ fixed. This is a systematic effect which we have not quantified at present, and so should be borne in mind when interpreting our current results. It will be necessary to analyse the dependence of our results on the level of chiral symmetry preservation by varying $L_{5}$ but at present this has not been performed.

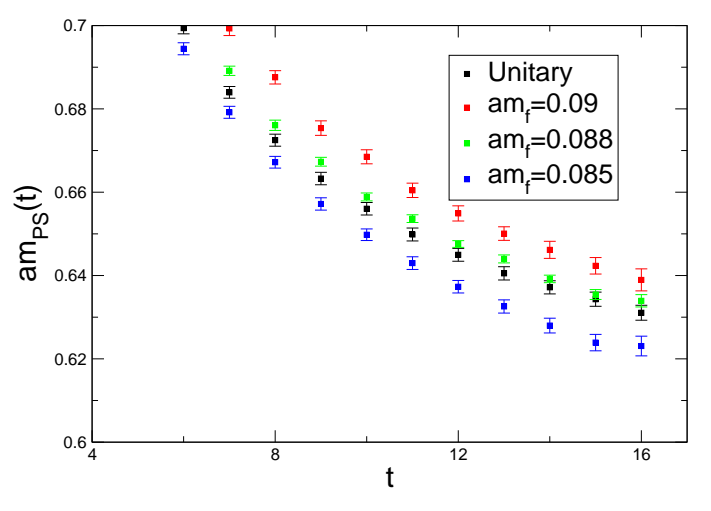

(a) Illustration of DWF tuning.

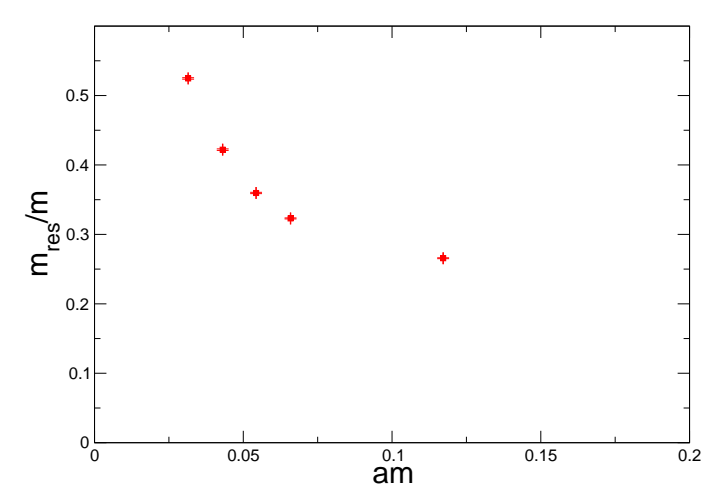

(b) Behaviour of $\frac{m_{\text {res }}}{m}$ with $m$.

Figure 1: Diagnostics on our simulation.

\section{Correlators}

We will compute the $S$ parameter contribution via the V-A expression (1.3) for which we 
need to compute the $\mathrm{VV}$ and $\mathrm{AA}$ vacuum polarisation functions. We will analyse the vacuum polarisations $\Pi^{\mathscr{J}}$ and $\Pi^{\mathrm{J}}$ which represent the conserved-local and local-local vector-vector and axial-vector-axial-vector correlators

$$
\begin{aligned}
& \Pi_{\mu v}^{\mathscr{J}}(\bar{q})=Z_{\mathrm{J}} \sum_{\mathbf{x}} e^{i q \cdot x}\langle\mathscr{J}(\mathbf{x}) J(0)\rangle \\
& \Pi_{\mu v}^{\mathrm{J}}(\bar{q})=Z_{\mathrm{J}}^{2} \sum_{\mathbf{x}} e^{i q \cdot x}\langle J(\mathbf{x}) J(0)\rangle
\end{aligned}
$$

where $\mathscr{J}(x)$ represents the conserved DWF vector or axial current and $J(x)$ the local DWF current. $Z_{\mathrm{J}}$ here is a renormalisation factor. In our calculation we rely on the equality $Z_{\mathrm{V}}=Z_{\mathrm{A}}$ which holds up to terms proportional to $m_{\text {res }}$. We caculate $Z_{\mathrm{V}}$ from the ratio of the conserved-local and locallocal vector two-point functions at zero momentum. From the $\Pi_{\mu v}$ we form the conserved-local and local-local $\mathrm{V}-\mathrm{A}$ vacuum polarisation tensors

$$
\begin{aligned}
\Pi_{\mu v}^{\mathscr{V}-\mathscr{A}} & =\Pi_{\mu v}^{\mathscr{V}}-\Pi_{\mu v}^{\mathscr{A}} \\
\Pi_{\mu v}^{V-A} & =\Pi_{\mu v}^{V}-\Pi_{\mu v}^{A}
\end{aligned}
$$

These tensors can be decomposed in their transverse and longitudinal components as

$$
\Pi_{\mu v}(\bar{q})=\left(\bar{q}^{2} \delta_{\mu v}-\bar{q}_{\mu} \bar{q}_{v}\right) \Pi_{\mathrm{T}}\left(\bar{q}^{2}\right)+\bar{q}_{\mu} \bar{q}_{v} \Pi_{\mathrm{L}}\left(\bar{q}^{2}\right)
$$

where $\bar{q}$ signifies the lattice momentum

$$
\bar{q}_{\mu}=\frac{2}{a} \sin \left(\frac{a q_{\mu}}{2}\right)=\frac{2}{a} \sin \left(\frac{\pi n_{\mu}}{L_{\mu}}\right)
$$

where $n_{\mu}$ is a 4 -vector of integers representing the discrete lattice momenta and $L_{\mu}$ is the 4-vector of integers representing the extent of the lattice in each of the space-time directions. The conservedlocal vector vacuum polarisation function obeys an exact Ward Identity

$\bar{q}_{\mu} e^{\frac{i q \mu a}{2}} \Pi_{\mu \nu}^{\mathscr{V}}=0$ which is equivalent to the vanishing of the longitudinal component of this polarisation tensor. In Figure 2a we show examples of the transverse component of the conserved-local and local-local $\Pi^{\mathrm{V}-\mathrm{A}}$. In Fig. $2 \mathrm{~b}$ we show how this function varies on ensembles with different masses and volumes.

\section{Results}

In order to extract the $S$ parameter contribution from our data, we must compute

$$
S=4 \pi \frac{d}{d(\bar{q})^{2}} \Pi_{\mathrm{T}}^{V-A}\left(\bar{q}^{2}\right)=4 \pi \frac{d}{d(a \bar{q})^{2}} a^{2} \Pi_{\mathrm{T}}^{V-A}\left(\bar{q}^{2}\right)
$$

where the last equality reassures us that we need not concern ourselves with fixing the scale in our simulation in order to compute the result we seek at on this single value of the coupling. We must also subtract from our result the alteration of the theoretical prediction for the $S$ parameter made by removing the Standard Model Higgs in building the complete model of DEWSB. To estimate this quantity we follow [7], finding that due to the fact that the mass splitting between the PS and 


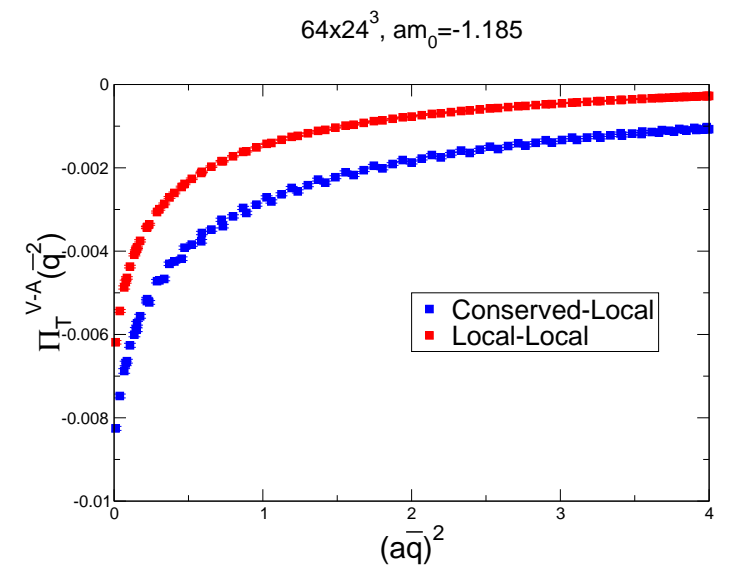

(a) Examples of the transverse component of the $V-A$ vacuum po-(b) larisation function.

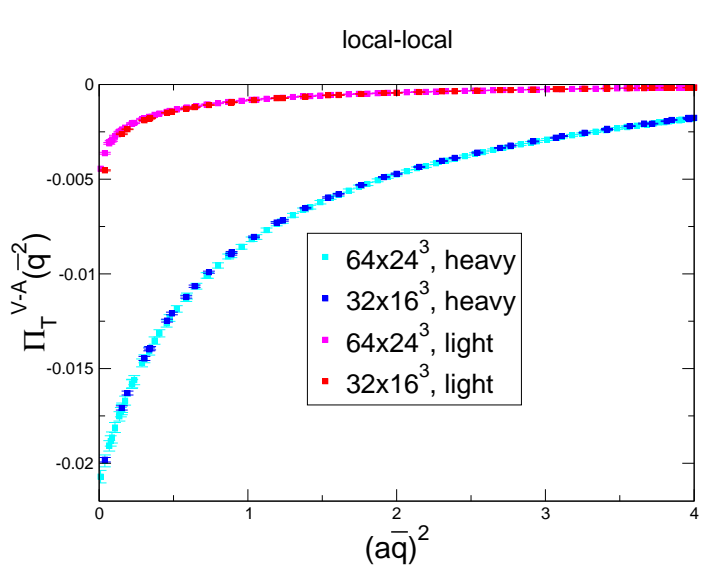

-(b) Further examples of the transverse vacuum polarisation from ensembles at different volumes and quark masses.

Figure 2: Examples of the transverse component of $V-A$.

$\mathrm{V}$ channels is heavily suppressed in this theory [8], the result is small relative to the scale and precision of our current results.

Our results for $S$ will depend on the slope of the transverse $V-A$ polarisation function, and so we must interepolate a continuous form for this function from our data. To do this we fit to the functional form using a series of Pade approximants, taking direction from similar techniques used to fit the vector polarisation function in order to extract the leading order hadronic contribution to the anomalous magnetic moment of the muon in QCD.

In order to analyse the stability of fits obtained using a given fit-form we will vary the data points included in the fit by fitting in the range $\left[0,\left(a \bar{q}_{C}\right)^{2}\right]$ and varying $\left(a \bar{q}_{C}\right)^{2}$. We estimate the systematic uncertainty owing to the uncertainty over how to perform this fit by choosing a range of results which are compatible with the results given by a number of different fit forms over a certain range of $\left(a \bar{q}_{C}\right)^{2}$. In Fig. 3a we show how the results typically depend on the fit ansatz used. We choose a range in $q_{\mathrm{C}}^{2}$ where the results are reasonably extracted from a number of different ansatze, and chose a final result for the slope at the origin which is compatible with all results obtained in this range.

Using this prescription, the results we obtain for the $S$ parameter are shown as a function of quark mass in Fig. 3b. These results are provisional and come with caveats, primarily that the chiral symmetry breaking is increasing in our calculation as we move to lighter quark masses, which might be expected to have a significant effect on our results. This effect will be explored in the near future. However at this point no suppression of the $S$ parameter in this theory is identified, nor do we identify any trend in the chiral limit.

\section{Conclusions}

We have obtained early provisional results for the $S$ parameter contribution from a MWT model of DEWSB. We will improve these results by resolving the dependence of the results on the 


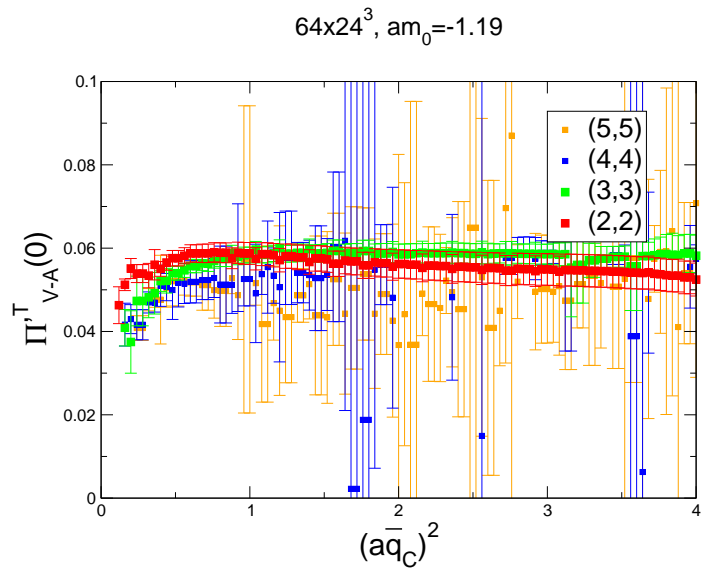

(a) Example of the varying results obtained from different Pade ansatze.

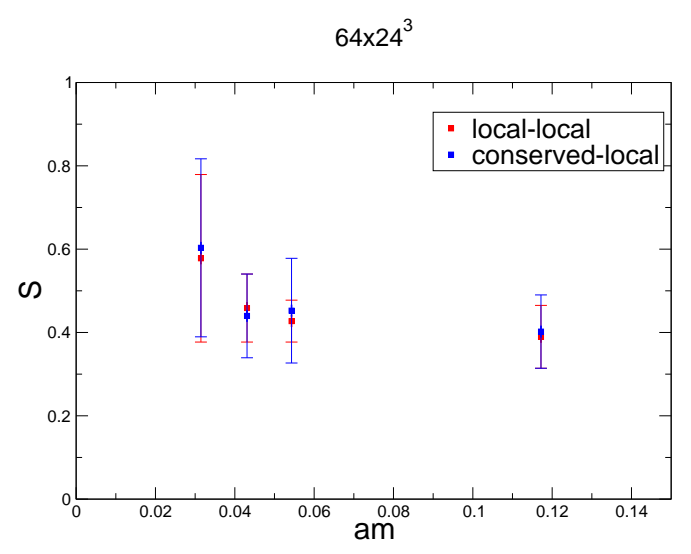

(b) Provisional results obtained for the $S$ parameter in MWT.

Figure 3: Results for $S$ obtained using our fitting procedure.

level of chiral symmetry breaking, and by improving the systematics of the study with regard to the tuning of the valence action, the renormalisation factors used, and the interpolation of the vacuum polarisation to zero momentum. In addition we will explore the consequences of alternating the order in which we take the zero momentum and chiral limits, as suggested in [10].

\section{References}

[1] E. Shintani et al. S-parameter and pseudo-Nambu-Goldstone boson mass from lattice QCD. Phys. Rev. Lett., 101:242001, 2008, 0806.4222.

[2] Peter A. Boyle, Luigi Del Debbio, Jan Wennekers, and James M. Zanotti. The S Parameter in QCD from Domain Wall Fermions. 2009, 0909.4931.

[3] Michael Edward Peskin and Tatsu Takeuchi. A New constraint on a strongly interacting Higgs sector. Phys. Rev. Lett., 65:964-967, 1990.

[4] Michael Edward Peskin and Tatsu Takeuchi. Estimation of oblique electroweak corrections. Phys. Rev., D46:381-409, 1992.

[5] K. Nakamura et al. Review of particle physics. J.Phys.G, G37:075021, 2010.

[6] Thomas Appelquist and Francesco Sannino. The Physical spectrum of conformal SU(N) gauge theories. Phys.Rev., D59:067702, 1999, hep-ph/9806409.

[7] Thomas Appelquist et al. Parity Doubling and the S Parameter Below the Conformal Window. Phys.Rev.Lett., 106:231601, 2011, 1009.5967.

[8] Luigi Del Debbio, Biagio Lucini, Agostino Patella, Claudio Pica, and Antonio Rago. Mesonic spectroscopy of Minimal Walking Technicolor. Phys. Rev., D82:014509, 2010, 1004.3197.

[9] Vadim Furman and Yigal Shamir. Axial symmetries in lattice QCD with Kaplan fermions. Nucl. Phys., B439:54-78, 1995, hep-lat/9405004.

[10] Francesco Sannino. Mass Deformed Exact S-parameter in Conformal Theories. Phys.Rev., D82:081701, 2010, 1006.0207. 